\title{
Super Star Cluster Luminosity Functions in Interacting Luminous Infrared Galaxies
}

\author{
Zara Randriamanakoto ${ }^{1,2}$ and Petri Väisänen ${ }^{2}$ \\ ${ }^{1}$ Astronomy Department, University of Cape Town \\ Private Bag X3, Rondebosch 7700, South Africa \\ email: zara@saao.ac.za \\ ${ }^{2}$ South African Astronomical Observatory, \\ P.O. Box 9, Observatory 7935, South Africa \\ email: petri@saao.ac.za
}

\begin{abstract}
Young and massive super star clusters (SSCs) are found whenever very active star formation is going on, such as that in interacting Luminous Infrared Galaxies (LIRGs). From a deep NIR adaptive optics imaging survey, we present thus far the first K-band SSC luminosity functions (LFs) in these types of galaxies, and also a relation between the brightest SSC and the global SFR of the galaxy. Based on the derived LFs, one can constrain the cluster initial mass function (CIMF) and study the formation and evolution of SSCs. Our preliminary results are in disagreement with theoretical expectations which suggest that the SSC LF should be well fitted by a single power-law with an index of -2 . We find power-law indexes of $\sim-1.5$, shallower than the expected ones. Taken at face value, our results appear to support the concept that the CIMF is mass-dependent, not universal, which will be studied in more detail by mass-modelling of the SSCs. The data-set will also allow us to estimate the fraction of total star formation originating in the SSCs over a range of galaxy types.
\end{abstract}

Keywords. galaxies: interactions, galaxies: star clusters, infrared: galaxies

\section{Introduction}

It is often stated that most stars are born in clusters (e.g. Lada \& Lada 2003), but much remains unclear on the early star cluster evolution, and hence galaxy evolution. Very massive $\left(10^{3}\right.$ to $\left.10^{7} M_{\odot}\right)$ young "super star clusters" SSCs, have been found in large numbers in strongly star-forming interacting galaxies (e.g. Whitmore et al. 1999). Can the most massive ones only form in those extreme conditions? How do the galaxy properties affect the outcome? What is their subsequent evolution and life, which ones survive and perhaps end up as (old) globular clusters? These questions are studied in our on-going survey of a few dozen strongly star-forming galaxies using the thus-far little utilized K-band near-IR Adaptive Optics (AO). Our NIR observations allow detection of much more obscured regions of the starbursts compared to the many mostly optical HST-based studies done so far.

Despite the significant work already done over the past decades, the early stages of evolution of SSCs still remain unclear. As a first step to analyze the SSC populations, we construct their luminosity functions, which can then be used to constrain e.g. the cluster initial mass functions and SSC formation and disruption models, as well as study the origin and propagation of star formation globally in the galaxies. 

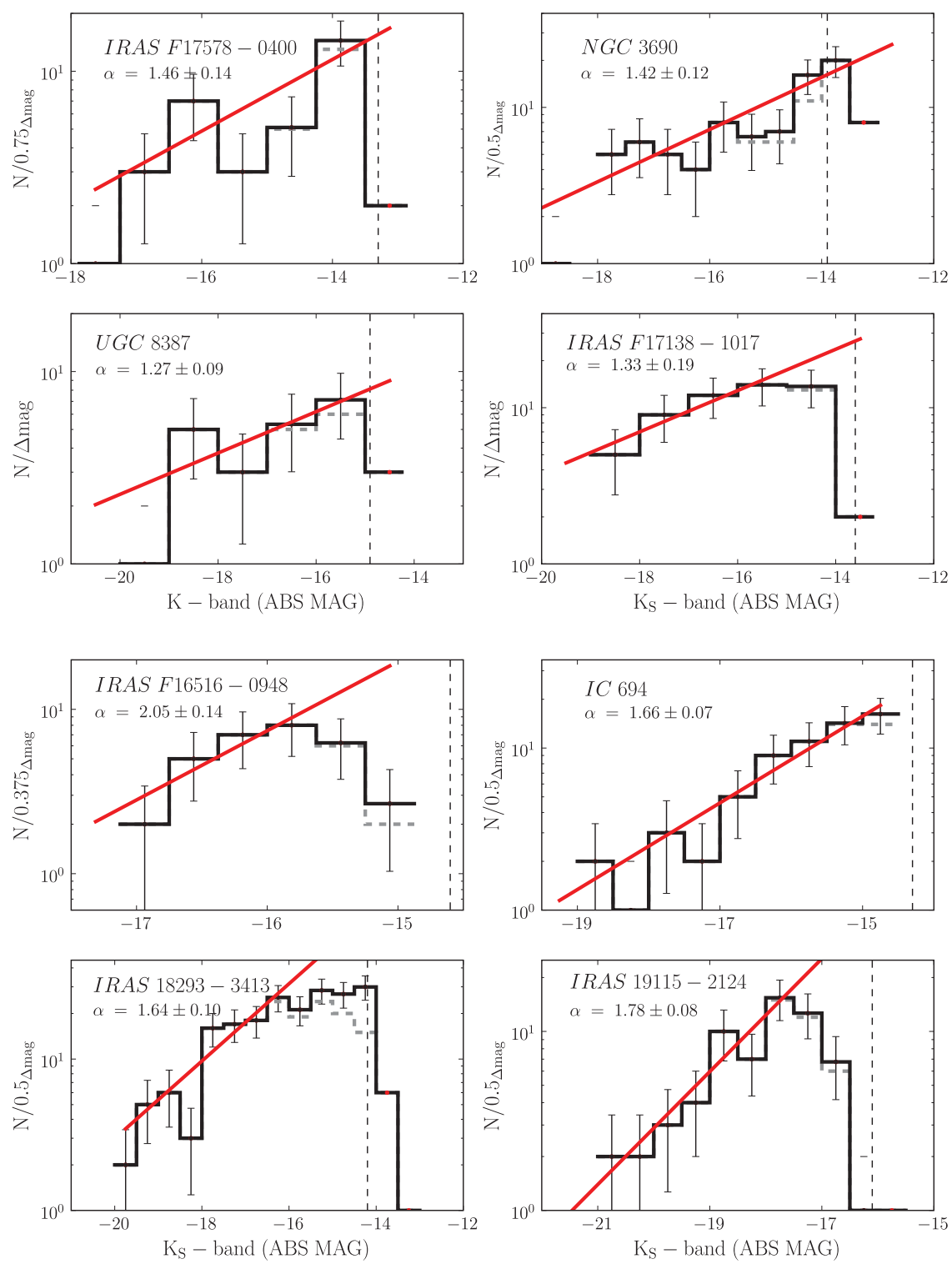

Figure 1. SSC candidate $\mathrm{K}_{S}$-band LFs using a constant bin size: the black solid histogram is the incompleteness corrected LF while the dashed curve shows the original raw counts. The vertical line marks the $50 \%$ completeness level. A weighted linear fit of the data points is overplotted (thick red straight line) with the LF. Note that the bin sizes of the sample are not the same, they are optimized for adequate statistics, and hence the y-axis label varies from one target to another.

\section{The sample}

The galaxies are at distances ranging from 40 to $180 \mathrm{Mpc}$, span the range from starbursts to high-luminosity LIRGs, and are at various stages of merging, interaction, or isolation. VLT/NACO is used for the main survey, while here we report on a pilot study using the Gemini/ALTAIR/NIRI instrument (Väisänen et al. 2008; Randriamanakoto 
et al. 2011, in prep). Both instruments deliver frames with a pixel scale of $\approx 0.02 \% /$ pixel and spatial resolution of $\approx 0.1$ ". The data are very well complemented with existing HST optical data for many of the targets.

\section{The NIR Luminosity Function}

We performed aperture photometry on point sources in the complex background conditions of the galaxies along with relevant completeness corrections and blending simulations. The resulting SSC candidate number counts are fitted with a power-law of the form $\Phi(L) d L \propto L^{-\alpha} d L$. The fitted power-law indexes are typically $\alpha \approx 1.6 \pm 0.2$ for our initial sample of galaxies (Fig. 1). This is significantly shallower than most slopes observed in the optical, which tend to be close to 2 (e.g. Elmegreen \& Efremov 1997). Our LF slope could perhaps indicate mass-dependent disruption of the SSCs, or environment and/or SFR dependent cluster-IMFs: most other determinations are from less luminous IR-galaxies. Interestingly, Adamo et al. (2010) recently found a similar shallow slope for the LIRG Haro 11. We note, also, that in a couple of cases (e.g. IRAS 18293-3413) a two-component power-law would give a better fit for the data, and the bright end slope would be steeper. We stress that these results will soon be checked with a significantly larger sample and (even) more careful photometric completeness and blending analysis and modelling.

\section{The Brightest SSC vs. SFR}

We also looked for a relation between the brightest K-band SSC vs. the SFR of the host galaxy (Fig. 2, which also includes the best fit equation of the relation). This is the first such relation derived for a NIR SSC sample, and is similar to equivalent relations in the optical (see e.g. Larsen 2002, Weidner 2004 and Bastian 2008). The reason for the relation could be related to the probability of the most massive clusters being formed in the most intense SF regions, and/or be a reflection of the current level of SFR where the youngest SSCs are the brightest. It is also interesting to note that comparing the slopes of the brightest SSC vs. SFR relations in the V-band (Bastian 2008) to that we found in the K-band, it is obvious that the $V-K$ colour of the brightest SSC reddens with SFR, suggesting more and more obscured environments for the most extreme star formation.

\section{Conclusions and on-going work}

The formation of massive star clusters remains poorly understood despite the amount of work which have been done over the past decades. It his pilot study we derive for the first time LFs and brightest cluster vs. SFR relations in the NIR for SSCs. The LF slopes are found to be surprisingly shallow, perhaps indicating target, or mass-dependent formation and disruption mechanisms for SSCs, thus contributing toward understanding the formation and the evolution of such star clusters.

To strengthen our NIR LF work, we are in the process of imaging a larger sample of southern LIRGs and starbursts with the VLT/NACO to find much more SSCs and to bring the number of host galaxies to $\sim 40$. In addition, we will estimate the ages and masses of the NIR detected SSCs with the help of archival HST data to be able to constrain models. Furthermore, we plan to extend the wavelength range of the observed sample to sub-mm and radio to study with high spatial resolution the gas disks and giant molecular clouds of these targets to understand the triggering of SSC formation in the turbulent interactions of LIRGs and starbursts. 


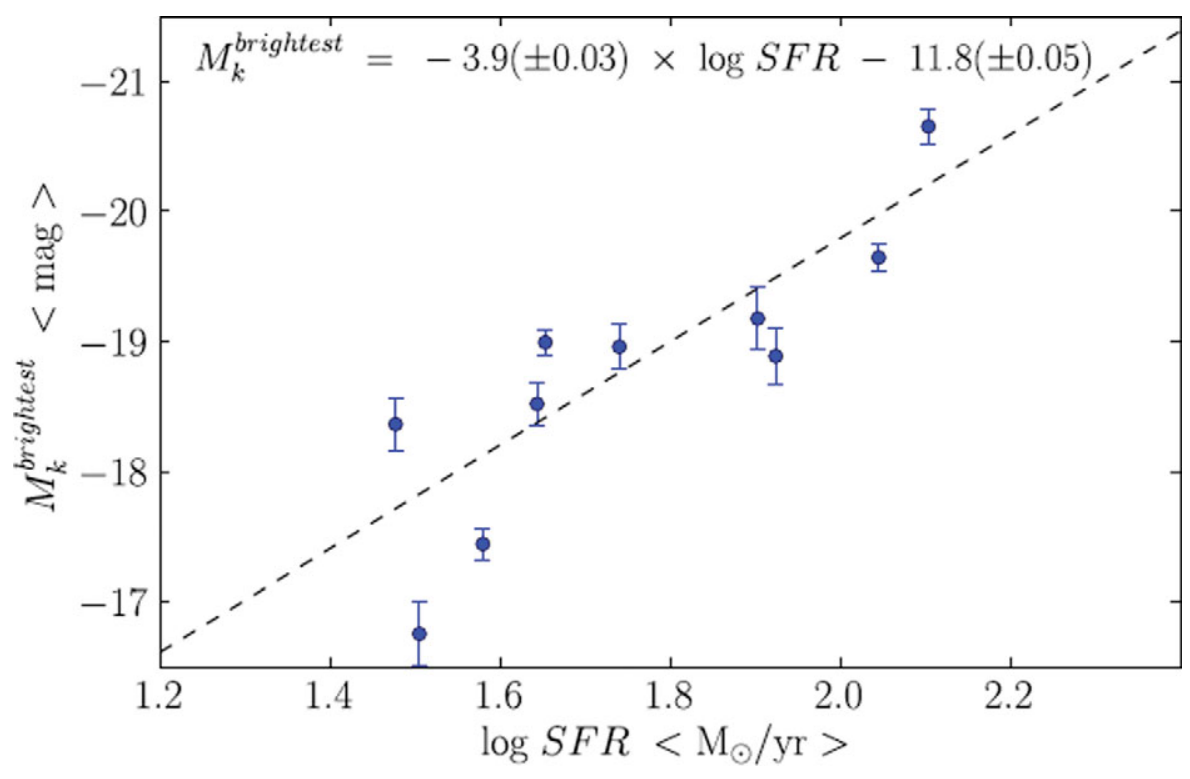

Figure 2. From observational K-band data, we were able to draw a relation between the brightest star cluster of the galaxy $M_{K}^{\text {brightest }}$ and its SFR. The dashed line represents a weighted linear fit to the data, and the equation at the top gives that exact relation.

\section{References}

Adamo, A. et al. 2010, MNRAS, 407, 870

Bastian, N. 2008, MNRAS, 390, 759

Lada, C. J. \& Lada, E. A. 2003, ARAA, 41, 57

Larsen, S. S. 2002, $A J, 124,1393$

Elmegreen, B. G. \& Efremov, Y. N. 1997, ApJ, 480, 235

Weidner, C., Kroupa, P., \& Larsen, S. S. 2004, MNRAS, 350, 1503

Whitmore, B. C. et al. 1999, AJ, 118, 1551

Väisänen, P. et al. 2008, MNRAS, 384, 886 